\title{
Lens-Free Optical Scanners for Metal Additive Manufacturing
}

\author{
CHARLES BIBAS $\oplus^{1,2}$ \\ 01.-Tecnica Inc., New York, NY 10018, USA. 2.-e-mail: cbibas@tecnica.com
}

Galvanometer scanners (GSs) driving selective laser sintering (SLS)/selective laser melting (SLM) printers for additive manufacturing (AM) have mechanical limits. They provide inconsistent energy density across the print surface because of changes in optical path length, surface beam speed, and angle of incidence. The resulting thermal gradients may be particularly problematic for metal, whose high heat conductivity makes temperature prediction during printing critical. In this paper, we mathematically analyze and compare GSs with a new lens-free optical scanner. The results show that the latter can facilitate metal printing by providing consistent energy deposition across the print surface.

\section{INTRODUCTION}

Galvanometer scanners (GSs) have been used for decades in SLM printing. The technology has been improved with better optomechanical control systems, better optics, better manufacturing capabilities, and even the use of machine learning. ${ }^{1-3}$ These incremental improvements have significantly improved the quality of metal parts, but its inherent limitations prevent further improvements.

As the laser beam scans the print surface, fundamental factors, viz. the surface beam speed, the optical path length, and the angle of the incident beam to the surface (which affect the beam size and shape), unavoidably vary. This means that two parameters that are critical for metal printing, i.e., the energy density and surface temperature, will vary and be difficult to control.

Commonly used measures of the energy density supplied by a moving laser beam include the surface energy density or Andrew number ${ }^{4,5}$

$$
\mathrm{Ed}=P_{1} /(v \cdot \text { Def })
$$

where $P_{1}$ is laser power, $v$ is scan speed, and Def is laser beam diameter or hatch width, and the volumetric energy density (Ev), which extends the Andrew formula to the volume of the top layer by including the layer thickness, $L_{h}$ :

$$
\mathrm{Ev}=P_{1} /\left(v \cdot \operatorname{Def} \cdot L_{h}\right)
$$

As is clear from these equations, if the beam speed, size, and shape vary across the print surface, the energy density will also vary and be difficult to control. The resulting thermal gradients are particularly problematic for metal, whose high heat conductivity makes temperature prediction during printing critical. Numerous studies such as those of Goodridge $^{4}$ and Bertoli ${ }^{6}$ suggest that Ev must remain within a narrowly defined range to maintain uniform melting of the powder. Nonuniform temperature will cause thermal stress to appear in the model, ${ }^{7}$ resulting in tensile residual stress on the surface and acceleration of crack propagation.

A new lens-free optical scanner (LFOS) has been developed by Tecnica to largely eliminate the current shortfalls of GS devices. The Øgon scanner maintains constant surface beam speed, constant optical path length, and an incident beam that is always perpendicular to the surface. It thus provides uniform energy density and uniform temperature across the print surface.

While many models have been developed to analyze and control the energy density and temperature distribution in AM metal printing, including those in Refs. 8-11, most consider the idealized case in which the energy input from the scanner is constant; they do not account for the inconsistency of GSs across the print surface. The Øgon, on the other hand, provides the uniform energy input that these models assume.

In this paper, we provide some mathematical analysis of the LFOS. The results show that it can

(Published online February 14, 2022) 
facilitate metal printing by providing consistent energy deposition across the print surface.

\section{METHODS}

The GS system is analyzed using the postobjective scanning method in which the scanner is positioned after the objective lens. The rays converge to be focused at the work surface even though the beam source is collimated. Choosing this method can expose weaknesses in the optical system in comparison with the preobjective method.

Although herein we analyze the GS and compare it to the Øgon, polygonal mirrors offer similar results to GS in that they deliver nonconstant values of OPL and as a function of beam location, thus the temperature prediction chart is similar to that obtained using GS.

The optomechanical properties of GS and Øgon scanners are analyzed mathematically and compared with regard to the main factors complicating temperature prediction, which is critical in AM of metal: beam speed, diameter, and shape, which affect Ev, and the beam thermal gradient.

Mathematical models are used to produce a system capability chart for each scanning system using which the pros and cons of each scanner are illustrated and compared.

\section{GALVANOMETER: ADVANTAGES AND LIMI- TATIONS}

Transfer functions represent the properties of the beam as an output, where the input is the command parameter(s). For the GS system, the transfer functions are a function of $\theta_{x}$ and $\theta_{y}$ (input), where $\theta_{x}=2 \cdot \omega \cdot t$ and $\theta_{y}=2 \cdot \omega \cdot t$, with $\omega \cdot t$ being the angular rotation of the respective motor (Fig. 1).

\section{Position}

The beam location can be described as ${ }^{12}$

$$
\begin{gathered}
y\left(\theta_{y}\right)=d \cdot \tan \left(\theta_{y}\right) \\
x\left(y, \theta_{x}\right)=\left(\sqrt{d^{2}+\mathrm{y}^{2}}+\mathrm{e}\right) \cdot \tan \left(\theta_{x}\right) \\
x\left(\theta_{x}, \theta_{y}\right)=\left(d \cdot \sqrt{1+\tan ^{2}\left(\theta_{\mathrm{y}}\right)}+\mathrm{e}\right) \cdot \tan \left(\theta_{x}\right)
\end{gathered}
$$

and the speed as ${ }^{12,13}$

$$
V x\left(\theta_{x}\right)=\frac{\mathrm{d} x}{\mathrm{~d} \theta_{x}}=\left(d \cdot \sqrt{1+\tan ^{2}\left(\theta_{\mathrm{y}}\right)}+\mathrm{e}\right) \cdot 1 / \cos ^{2}\left(\theta_{x}\right)
$$

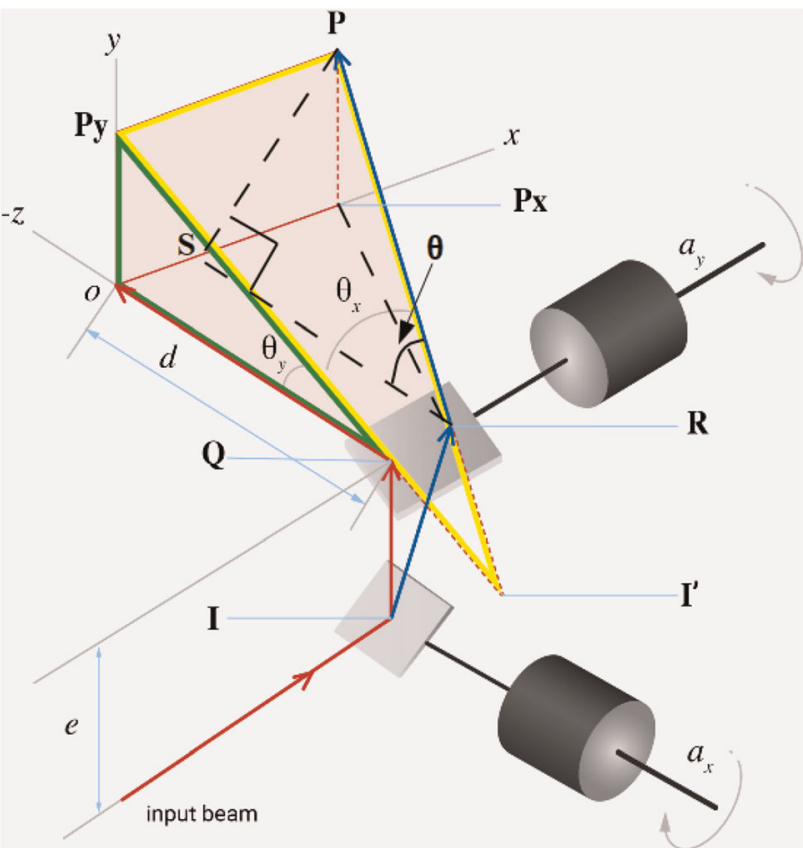

Fig. 1. GS optical scanner composed of two galvanometers in a $2 D$ scanning setting.

$$
V y\left(\theta_{y}\right)=\frac{\mathrm{d} y}{\mathrm{~d} \theta_{y}}=d \cdot \frac{1}{\cos ^{2}\left(\theta_{y}\right)}
$$

$$
V=\sqrt{\frac{\left(d \cdot \sqrt{1+\tan ^{2}\left(\theta_{\mathrm{y}}\right)}+\mathrm{e}\right)^{2}}{\cos ^{4}\left(\theta_{x}\right)}+\frac{\mathrm{d}^{2}}{\cos ^{4}\left(\theta_{\mathrm{y}}\right)}}
$$

\section{Optical Path Length}

The OPL is the path length from the beam source or a reference point on the optical axis to the incident point on the work surface, expressed as a function of $\theta_{x}$ and $\theta_{y} \cdot{ }^{13}$

$$
\mathrm{OPL}=\left(d \cdot \sqrt{1+\tan ^{2}\left(\theta_{\mathrm{y}}\right)}+\mathrm{e}\right) \cdot \sqrt{1+\tan ^{2}\left(\theta_{\mathrm{x}}\right)}
$$

\section{Angle of Incidence}

$\Theta$, the angle of incidence, is the angle between the incident beam and the normal to the surface. It is calculated from the triangle $P P x R$ as

$$
\Theta=\arccos \frac{d}{\sqrt{x^{2}+\left(\sqrt{\mathrm{d}^{2}+\mathrm{y}^{2}}+\mathrm{e}\right)^{2}}-e / \cos \left(\theta_{x}\right)}
$$


Alternatively, it can be expressed as a function of $\left(\theta_{x}, \theta_{y}\right)$ as

$$
\Theta=\arccos \frac{1}{\left[\sqrt{1+\tan ^{2}\left(\theta_{\mathrm{y}}\right)}+\frac{\mathrm{e}}{\mathrm{d}}\right] \cdot \sqrt{1+\tan ^{2}\left(\theta_{\mathrm{x}}\right)}-\frac{e}{d \cdot \cos \left(\theta_{x}\right)}}
$$

Equations 9 and 11 shows that $\Theta$ changes across the scan area. $\Theta$ is also one of the parameters affecting the beam intensity.

The OPL is a product of two nonlinear functions that change quickly as the beam moves away from the origin (print center). The OPL is also one of the parameters affecting the beam's focus and therefore its intensity.

\section{Symmetry}

It is evident from Eq. 3 that there is symmetry about the origin for GSy, where $y\left(-\theta_{y}\right)=-y\left(\theta_{y}\right)$. Similarly, from Eq. 4, GSx is symmetrical when $\theta_{y}$ is held constant where $x\left(-\theta_{x}, \theta_{0}\right)=-x\left(\theta_{x}, \theta_{0}\right)$ for $\theta_{y}=$

are the local coordinates of the image lying on the work surface. Beam augmentation is analyzed in two stages, wherein stage 1 is the effect due to OPL and stage 2 is the effect due to $\Theta$. The two stages can be quantified as follows:

The beam radius change as a result of OPL (stage $1)^{13}$ is

$$
r 1=r 0\left(\left(d \cdot \sqrt{1+\tan ^{2}\left(\theta_{\mathrm{y}}\right)}+\mathrm{e}\right) \cdot \sqrt{1+\tan ^{2}\left(\theta_{\mathrm{x}}\right)}-(d+\mathrm{e})\right) \cdot \mathrm{Ha}
$$

The beam cross-section grows symmetrically along both local axes, resulting in a larger circular beam diameter.

The beam radius change as a result of (stage 2$)^{13}$ (where only the local vertical axis changes), can be expressed as

$$
r_{2 v}=r_{0} \cdot \frac{1}{\cos (\Theta)}
$$

$$
r_{2 v}=\left(\left(\sqrt{1+\tan ^{2}\left(\theta_{\mathrm{y}}\right)}+\mathrm{e} / \mathrm{d}\right) \cdot \sqrt{\tan ^{2}\left(\theta_{x}\right)+1}-e /\left(d \cos \left(\theta_{x}\right)\right)\right) \cdot\left(r 0+\left(\left(\mathrm{d} \cdot \sqrt{1+\tan ^{2}\left(\theta_{\mathrm{y}}\right)}+\mathrm{e}\right) \cdot \sqrt{1+\tan ^{2}\left(\theta_{\mathrm{x}}\right)}-(\mathrm{d}+\mathrm{e})\right) \cdot \mathrm{Ha}\right)
$$

$\theta_{0}$. Note also that $x$ is a function of the two inputs $\theta_{x}$ and $\theta_{y}$. However, because the two GSs are positioned at different distances from the work surface (as the distance between GSx and GSy is $e$ ), this introduces asymmetry between the two axes.

Equations (3) and (4) show that

$$
y\left(\theta_{0}\right) \neq x\left(\theta_{0}\right) \text { for } \quad \theta_{y}=\theta_{x}=\theta_{0}
$$

Additionally, it is evident from Eqs. 6 and 7 that

$$
V_{x}\left(\theta_{0}\right) \neq V_{y}\left(\theta_{0}\right) \text { for } \theta_{y}=\theta_{x}=\theta_{0}
$$

This asymmetry and nonlinear speed make it more difficult for the two-GS system controller to maintain the constant speed required to keep Ev within the narrow target range.

\section{Beam Size and Shape}

Consider a circular beam with radius $r_{0}$ and at focus at the origin; as the beam moves across the work surface, the beam size and shape will change as $\Theta$ and OPL change.

As the beam traverses the surface and deviates from zero, the beam's image on the surface stretches from a circle to an ellipse, whose vertical and horizontal axes we denote by $r_{v}$ and $r_{h}$, where $v$ and $h$

$$
r_{2 h}=r_{1}
$$

$$
A=\pi \cdot r_{2 h} \cdot r_{2 v}=\pi \cdot r_{1}^{2} \cdot \frac{1}{\cos (\Theta)}
$$

substituting 1/cos $(\Theta)$ with Eqs. 10 and 11 yields

$$
\begin{aligned}
A= & \pi \cdot\left(r 0+\left(\left(\mathrm{d} \cdot \sqrt{1+\tan ^{2}\left(\theta_{\mathrm{y}}\right)}+\mathrm{e}\right) \cdot \sqrt{1+\tan ^{2}\left(\theta_{\mathrm{x}}\right)}-\right.\right. \\
& (d+\mathrm{e}) \cdot \mathrm{Ha}))^{2} \cdot\left(\left(\sqrt{1+\tan ^{2}\left(\theta_{\mathrm{y}}\right)}+\frac{\mathrm{e}}{\mathrm{d}}\right) \cdot \sqrt{1+\tan ^{2}\left(\theta_{\mathrm{x}}\right)}-\frac{e}{d \cdot \cos \left(\theta_{x}\right)}\right)
\end{aligned}
$$

It is apparent from Eqs. 16 and 17 that the energy density $E / A$ changes as the beam moves across the work surface. Moreover, Eqs. 16 and 17 is not symmetrical with respect to $\theta_{x}$ and $\theta_{y}$, and for $x, y$ coordinates:

$$
\begin{gathered}
y\left(\theta_{x}\right) \neq x\left(\theta_{y}\right) \\
A(x, y) \neq A(y, x)
\end{gathered}
$$

Most importantly, the $\tan \left(\theta_{x}\right)$ and $\tan \left(\theta_{y}\right)$ components change nonlinearly as the angles grow. This 
causes sharper growth in $A$ as $\theta_{x}$ and $\theta_{y}$ grow. The asymmetry in $A$ augmentation needs to be considered for each pixel. Therefore, the modulator needs to be configured to take into account these nonlinearities and asymmetry. This also means that part positioning on the $x-y$ bed matters.

Furthermore, the speed transfer function is not symmetrical: $V_{x}\left(\theta_{0}\right) \neq V_{y}\left(\theta_{0}\right)$ for $\theta_{x}=\theta_{y}=\theta_{0}$. This further highlights the asymmetry in energy deposition as the beam speed is one of the parameters dictating the energy density absorbed by the surface.

\section{Linearity}

It is apparent from Eqs. 3 and 4 that the transfer function for either $x$ or $y$ is nonlinear. The use is limited to small angles where $\tan \left(\theta_{x}\right)$ or $\tan \left(\theta_{y}\right)$ is close to linear. It is evident from Eqs. 10 and 11 that $e$, the distance between GSx and GSy, must be kept as small as possible in relation to $d$, the distance between GSy and the work surface. The smaller the value of $e / d$, the smaller the asymmetry around all axes.

\section{Modulation}

Modulation in a GS system is performed using a closed loop. The challenges lie in addressing nonlinear transfer functions (position, speed, and energy deposition for printing) and the mechanical inertia of the oscillatory element in the GS. The nonlinear positioning can be addressed by geometric correction techniques. ${ }^{14}$ Similarly, Zhang ${ }^{15}$ further measured data spots and performed online calibration of the GS.

Duma $^{16}$ further explored the GS mechanical inertia of the oscillatory element limitation. He qualifies the modulation speed limit to be around $1.5 \mathrm{kHz}$ and selected the preferred/optimized modulation waveform to be a sawtooth wave with fast and slow-moving GS where the raster method is used.

\section{Offset/Drift Errors}

Offset/drift errors occur when the GS deviates from the origin and as a result moves all respective coordinates. Offset/drift errors are temperature sensitive and must be watched closely. Offset/drift errors can be addressed by online correction on the fly, in a similar method to Zhang. ${ }^{15}$

Offset errors are measured in microradians. A typical value from a high-quality GS manufacturer is less than 10 microradians. Some manufacturers, unfortunately, amplify the offset/drift error when they make design choices to reduce -related errors. As an example, a company may enlarge $d$ (the distance from the GSy to the work surface) from 500 $\mathrm{mm}$ to $2000 \mathrm{~mm}$. Such enlargement of $d$ will reduce the maximum $\Theta$ required for the same size work surface, thereby reducing $\Theta$-related errors, but will also amplify offset/drift errors.

Consider a $0.1 \mathrm{~mm}$ beam diameter using a hash (grid) of $0.1 \mathrm{~mm}$. The position is now dictated by a longer arm $d$. A typical $5 \mu \mathrm{rad}$ offset/drift error will result in a $10 \mu \mathrm{rad}$ error in the correspondent GS $\theta$.

If the intended location is $x_{0}$, then the new location is

$$
x_{0} \pm \operatorname{Err}=x_{0} \pm d \cdot 10 \mu \mathrm{rad}=x_{0} \pm 20 \mu \mathrm{m}
$$

This is already a change of $20 \%$ of the grid size. Temperature changes will amplify this error by a factor proportional to the temperature change. As an example, Cambridge Technology for their GS model $83 \times x K$ spec reports a zero drift parameter of 5 $\mu \mathrm{rad} /{ }^{\circ} \mathrm{C}$. A change of $7{ }^{\circ} \mathrm{C}$ will thus result in a position change (for $d=2000 \mathrm{~mm}$ ) of

$$
x_{0} \pm \operatorname{Err}=x_{0} \pm 5 \cdot 2 \cdot 10^{6} \cdot 7 \mu \mathrm{rad}=x_{0} \pm 70 \mu \mathrm{m}
$$

Note also that this error is only for one GS. Adding the second GS vector will result in a total grid shift of $\pm \operatorname{sqrt}(2) \cdot 70 \mu \mathrm{m} \rightarrow \pm 99 \mu \mathrm{m}$.

\section{Repeatability}

The repeatability error is measured in units of microradians. It is doubled when reflecting from the GS mirror. Additionally, the error is further augmented when GSx and GSy errors are combined. A typical value for one GS is $5 \mu \mathrm{rad}$ to $10 \mu \mathrm{rad}$.

\section{Energy Density}

In this section, we calculate the volumetric energy density Ev (Eq. 2) as a function of the angle of incidence, taking into account the changes in beam speed and beam diameter discussed above, as well as Lambert's cosine law.

Define OPL' as the optical path length from the GSy axis to the work surface, where $d$ is the beam distance at focus for a beam converging in Ha. In this way, we can simplify the calculations without using $\theta_{y}$ or $\theta_{x}$ :

$$
\mathrm{OPL}^{\prime}=d / \cos (\Theta)
$$

Ha can be expressed with $M$ the beam size at the mirror and $d$ (Fig. 2):

Since $\operatorname{Def}_{0} \ll M$, we can simplify the term for $M$ as

$$
M=\operatorname{Def}_{0}+2 \cdot \mathrm{d} \cdot \mathrm{Ha} \rightarrow \mathrm{Ha}=\mathrm{M} /(2 \cdot \mathrm{d})
$$

Considering stage 1 beam enlargement and substituting $\mathrm{Ha}$, we have

$\operatorname{Def}_{1 h}=\operatorname{Def}_{1 v}=\operatorname{Def}_{1}=\operatorname{Def}_{0}+2 \cdot\left(\mathrm{OPL}^{\prime}-\mathrm{d}\right) \cdot \mathrm{M} /(2 \cdot \mathrm{d})$ 


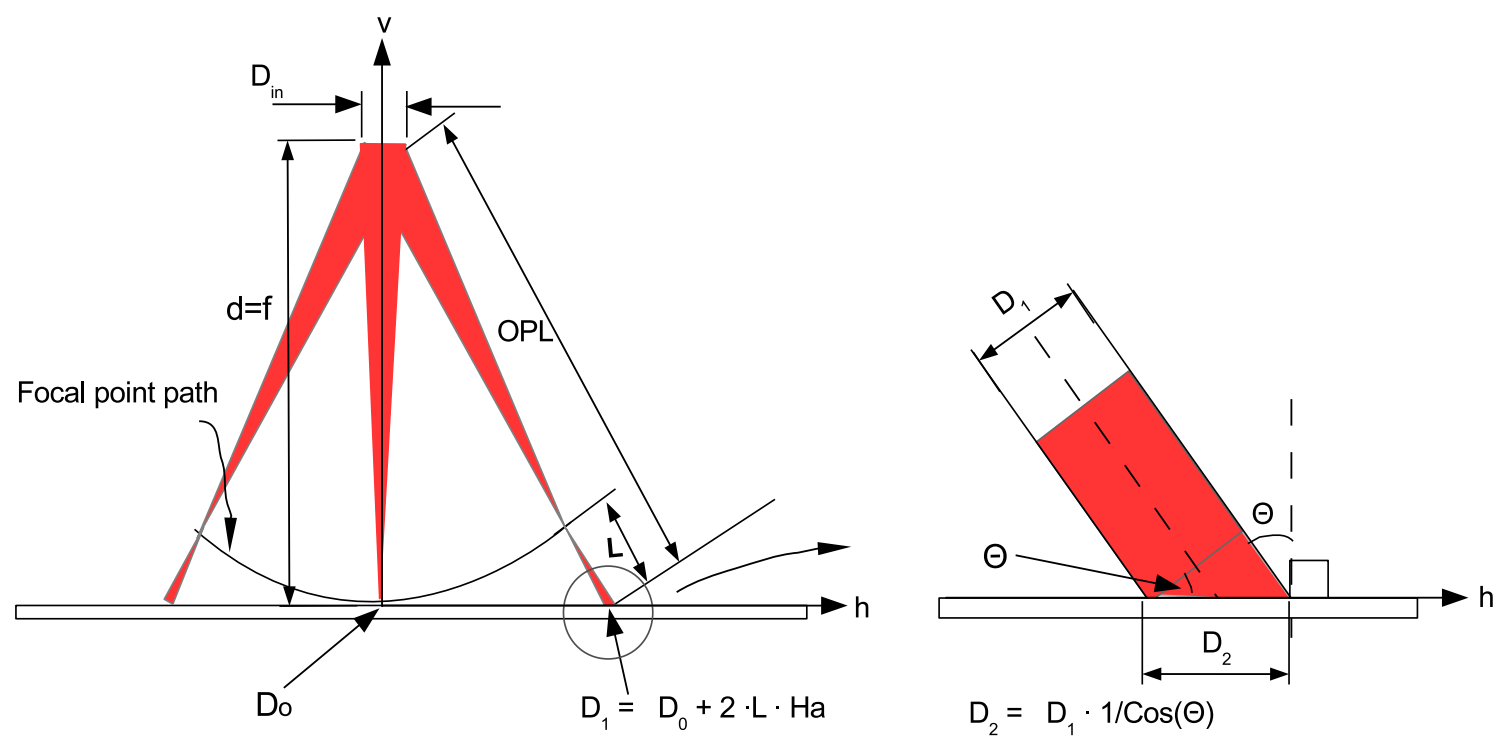

Fig. 2. Stage 1 and stage 2 beam enlargement.

$$
\operatorname{Def}_{1}=\operatorname{Def}_{0}+(1 / \cos (\Theta)-1) \cdot M
$$

Stage 2 beam enlargement affects only the vertical axis, thus

$$
\operatorname{Def}_{2 v}=\operatorname{Def}_{1} / \cos (\Theta)
$$

where Def $\mathrm{D}_{2 h}$ remains unchanged. We can now calculate a new Def for the two stages combined by calculating the area of the ellipse, then normalizing it to an equal circular area, resulting in

$$
\text { Def }=\frac{1}{\sqrt{\cos (\Theta)}} \cdot \operatorname{Def}_{1}=\frac{1}{\sqrt{\cos (\Theta)}} \cdot\left(\operatorname{Def}_{0}+\left(\frac{1}{\cos (\Theta)}-1\right) \cdot M\right)
$$

The beam speed is extracted from Fig. 2 as

$$
v=\mathrm{d} h / \mathrm{d} \Theta=\frac{d(d \cdot \tan (\Theta))}{d \Theta}=\frac{d}{\cos ^{2}(\Theta)}
$$

Using the Ev in Eqs. 2, 22 and 23 and applying Lambert's cosine law, this yields (Fig. 3)

$$
\mathrm{Ev}=\frac{P_{1}}{v \cdot \operatorname{Def} \cdot L_{h}}=\frac{P_{1} \cdot \cos (\Theta)}{\frac{2 \cdot R \cdot f \cdot d}{\cos ^{2}(\Theta)} \cdot \operatorname{Def} \cdot L_{h}}=P_{1} \cdot \frac{\cos ^{3}(\Theta)}{L_{h} \cdot v_{0} \cdot\left(\frac{\operatorname{Def}_{0}+\left(\frac{1}{\left(\frac{1}{\cos (\theta)}-1\right) \cdot \mathrm{M}}\right.}{\sqrt{\cos (\Theta)}}\right)}
$$

$$
\mathrm{Ev}=\frac{P_{1}}{L_{h} \cdot V_{0}} \cdot \frac{\cos ^{3}(\Theta) \cdot \sqrt{\cos (\Theta)}}{\left(\operatorname{Def}_{0}+\left(\frac{1}{\cos (\Theta)}-1\right) \cdot \mathrm{M}\right)}
$$

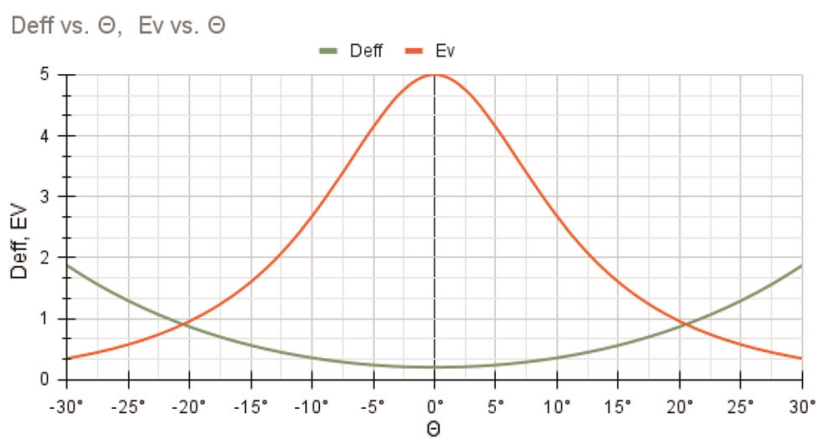

Fig. 3. Def and $E_{v}$ using $M=10 \mathrm{~mm}$ and $D_{0}=0.2 \mathrm{~mm}$, normalized for $P_{\mathrm{l}} /\left(L_{h} \cdot V_{0}\right)=5$.

\section{Temperature Prediction}

The energy deposition across the work area depends mostly on and the print path. Equations 25 and 26 shows that the energy deposition for each voxel depends on the voxel location, so the energy deposition varies as the beam moves across the print surface. Consider the case where the beam is moving away from the origin from voxel $n$ to voxel ( $n$ +1 ); the elongation of the beam shape on the surface means that, while the beam is activated for voxel $n$, voxel $(n+1)$ will be preheated. In contrast, when the beam is moving towards the origin, the next voxel is not preheated. This asymmetry further contributes to the nonuniform temperature distribution. Mirkoohi ${ }^{7}$ presented a model for temperature prediction where the laser source is normal to the print surface, moving in a straight line, emitting constant power, and moving with constant velocity. Mirkoohi solved the model for the temperature gradient at any $x, y$ location, resulting in Eq. 27, where the model captures the melt pool geometry for $x, y$. In her research, Mirakoohi also uses a 
numerical analysis model where both models are compared with experimental data.

Equation (27) further confirms that $T_{0}$, the initial voxel temperature, is one of the parameters affecting the gradient temperature. Therefore, a higher $T_{0}$ will introduce a larger temperature gradient due to the post heat when the beam moves away from the center compared with a voxel in the same location when the beam moves towards the center.

For the temperature prediction equation to obtain $T$ (the temperature gradient) at a distance $R$ from the laser spot where $R=\sqrt{x^{2}+y^{2}}$, Mirkoohi ${ }^{7}$ (for a laser moving along the $x$-axis) uses

$$
T=\frac{P_{1}}{4 \pi K R} \exp \left(\frac{-v(R-x)}{2 \propto}\right)+\mathrm{T}_{0}
$$

where $P_{1}$ is the laser power, $v$ is the laser velocity, $K$ is thermal conductivity, and $\alpha$ is thermal diffusivity.

\section{Attempts to Fix the Transfer Functions of the GS in AM}

An $\mathrm{f}$-theta lens is needed to keep the laser beam in focus on the flat plane of the work surface and to compensate for the speed variations across the surface. The speed is kept constant by changing the laser beam angle so that the beam distance traveled along the planar work surface is linearly proportional to the input rotation angle of the beam. More specifically, the f-theta lens transforms the beam direction from the entry angle $\theta$ to an exit angle $\arctan (\theta)$.

To also correct the angle of incidence and maintain a circular beam spot shape, a telecentric f-theta lens is required, and it must be at least as large as the work surface.

f-theta lenses are not perfect solutions and are not scalable. They are expensive, difficult to manufacture, have errors due to manufacturing tolerances, and cannot in practice be manufactured beyond a size limit.

\section{Conclusions for GS}

Although the GS provides 2D scanning in the $x$ and $y$ dimensions. the asymmetry and nonlinearity in positioning and speed make it more challenging to modulate when high-resolution, high-quality print/scanning is needed. This is especially true for $\mathrm{AM}$, where the analog intensity level is important, in contrast to sensing.

Although the modulation of the $y$ coordinate is dependent only on the $\theta_{y}$, modulation, the $x$ coordinate depends on both $\theta_{x}$ and $\theta_{y}$. Therefore, the $x$ modulator needs additional input. This puts a burden on the modulator when rendering a vector because the print path must be calculated to produce the outputs for each GS. Additionally, the beam cross-section changes with $\Theta$, resulting in additional degradation of the absorbed energy at the surface.
Attempts have been made to mitigate the GS positioning by Xianyu ${ }^{14}$ and the drift in situ by Zhang. ${ }^{15}$

The Mirkoohi model ${ }^{7}$ is based on a beam normal to the surface and constant beam speed, beam shape, and size, where the substrate is at the same temperature and the beam follows a straight-line path. Even if the GS uses a straight-line print path, the input parameters to the model will deviate from the Mirkoohi model, suggesting a more complex model where the heat flux emitted by the laser is broken down into two components: a vertical component and a horizontal component, both of which change in size and direction as $\Theta$ changes.

Additionally, the GS beam incident will vary by voxel location. Moreover, the beam is not normal to the surface, so melt pool models, such as that of Mirkoohi, ${ }^{7}$ do not fully reflect the GS operation, Therefore, the melt pool model is difficult to predict.

The heat deposition will vary for each voxel because of the numerous variables. Overall, the sum of all the voxels' temperature contributes to the layer temperature prediction.

\section{LFOS: Advantages and Limitations}

The Øgon LFOS consists of two reflectors (M1 and M2; Figs. 4, 5, 6), where M1 is a rotating reflector and M2 is an annular stationary reflector. The beam source strikes M1 along the optical axis, which is also the actuator rotational axis. The beam then travels towards M2, which diverts the beam to the work surface.

Because M2 is a circular ring of radius $R$, it imposes a focus at a distance $d=R / 2$ for a sphere and $R$ for a conical ring on the $x$ dimension of the beam (Fig. 5). The $y$ component of the beam is focused by the M1 $y$ surface component, and the M2 profile with the focus is selected to match the $x$ dimension distance at $R / 2$ from $\mathrm{M} 2$.

The LFOS functions performed on the beam source are:

1. Beam steering: first, it is steered by M1 from the beam source optical axis, then by M2 back parallel to the beam source optical axis;

2. Beam revolution around the beam source: the output beam revolves around the beam source optical axis;

3. Beam rotation around its local optical axis;

4. Beam focusing: the beam is fully focused in both directions at the worksurface.

The LFOS focuses a diverging beam without the use of lenses. As an example, a beam source from a multimode fiber with $105 \mu \mathrm{m}$ cladding diameter and NA0.15 focuses back to the beam source size in the Øgon first prototype, where $r=125 \mathrm{~mm}$ and $d=60$ $\mathrm{mm}$.

Because the beam rotates around its own axis, it radiates more consistent energy. 

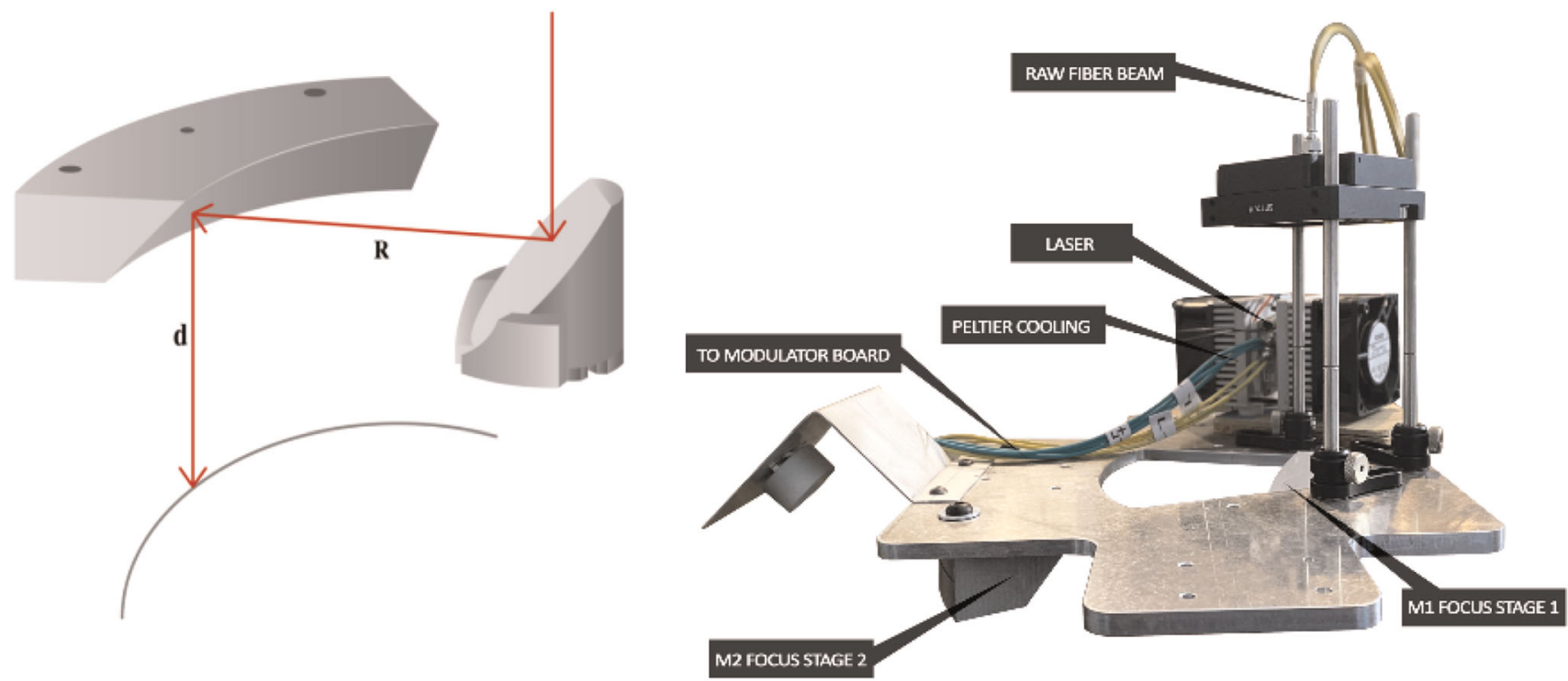

Fig. 4. Øgon and a printhead.

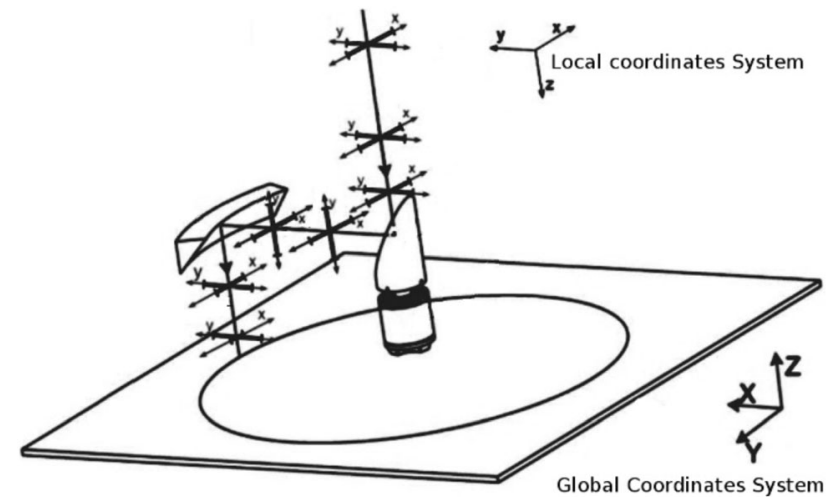

Fig. 5. Øgon optical functionality ${ }^{17,18}$.

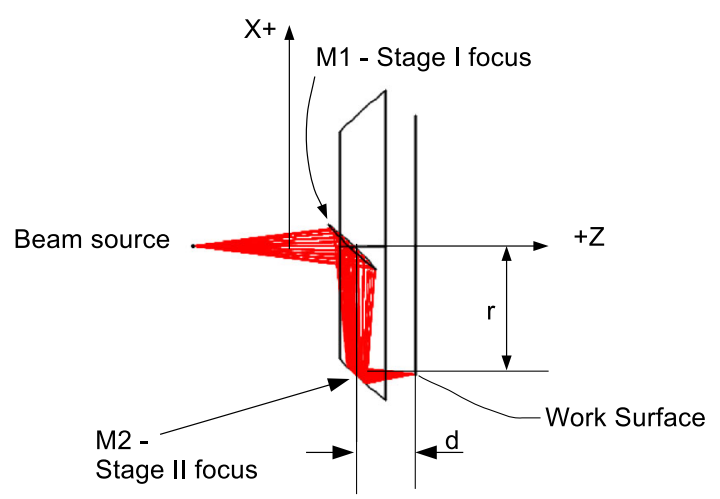

Fig. 6. Øgon working principle.

\section{Scanning Method}

LFOS uses a modified raster scanning method in which the $x$-axis is scanned in arcs instead of horizontal lines. After each arc, a linear conveyor moves the LFOS a fixed vertical distance along the $y$-axis to scan the next arc. This method resembles polygonal mirror (PM) two-dimensional (2D) printing. However, the result is greatly superior to the PM method because the PM must print on a cylindrical conveyor to keep the focus, while the LFOS is at focus printing on a flat surface. Additionally, the slow-moving conveyor wear and tear is very small compared, for example, with a CNC actuator because there is very little travel time during the print.

\section{Maximum Scanning Speed}

The rotational speed at the work surface is controlled by the motor rotational speed (rotations per second $f$ ). When $\mathrm{M} 1$ is carried by a brushless motor, $f=2000 \mathrm{~Hz}$ (2000 rotations per second). When $R=$ $125 \mathrm{~mm}$ is selected, the surface print speed is

$$
2 \cdot \pi \cdot R \cdot f=1570 \mathrm{~m} / \mathrm{s}
$$

For comparison, the maximum print speed of a GS is below $10 \mathrm{~m} / \mathrm{s}$.

\section{Position}

Since the beam is perpendicular to the work surface, the beam path at the work surface follows the radius curvature $R$ of M2. The beam location along each arc can be expressed simply using polar coordinates as follows:

The beam location $\theta$ where $r$ is constant, arc length $s(\theta)=\theta \cdot R$ or

$$
s(t)=\omega \cdot t \cdot R=2 \cdot \pi \cdot R \cdot f \cdot t
$$


Because the Øgon is carried by a linear conveyor between arcs, the beam position on the work surface in Cartesian coordinates can be expressed as

$$
y(t)=Y c(t)+\mathrm{R} \cdot \cos (2 \cdot \pi \cdot \mathrm{f} \cdot \mathrm{t}) \quad \mathrm{Yc} \text { is the conveyor location }
$$

$$
x(t)=R \cdot \sin (2 \cdot \pi \cdot f \cdot t)
$$

Alternatively, the beam location can be expressed with $i$ and $j$

$$
\begin{gathered}
y(i, j)=i \cdot \Delta y+\mathrm{R} \cdot \cos (2 \cdot \pi \cdot \mathrm{j} / \mathrm{P}) \\
x(j)=R \cdot \sin (2 \cdot \pi \cdot j / P)
\end{gathered}
$$

where $y$ is the interval to move from the current position. $\Delta y$ also represents the distance between the $\operatorname{arcs}, i$ is the arc number, and $j$ is the pixel location in the arc where $j=0$ when $x=0 . P$ is the number of pixels in a full circle.

\section{Beam Speed}

When the beam is activated, the linear conveyor is not moving. Therefore, the surface speed is calculated as

$$
v(t)=d s / d t=2 \cdot \pi \cdot R \cdot f
$$

where $s$ is the arc travel length.

\section{OPL}

There is no change in OPL, hence it is

$$
2 \cdot(d+\mathrm{R})
$$

where $d$ is the distance from M2 to the work surface and $r$ is the distance between M1 and M2.

\section{$\Theta$}

The beam is always normal to the work surface, $\Theta$ $=0^{\circ}$.

\section{Beam Size and Shape}

As the beam is in focus and perpendicular to the work surface at any M1 angular position and at any position of the linear conveyor, the size and shape of the beam remain constant. Hence the beam radius never varies from $r_{0}$.

\section{Modulation}

Modulation is performed open loop because the rotational speed is constant. The inertia of the rotating, balanced M1 helps keep the speed stable and undisturbed.

LFOS printheads scan in a series of arcs. The radius of each arc is $R$, the distance between M1 and
M2. As the rotational speed is constant, the locations of the pixels in each arc are dictated by a constant time interval between them. This direct modulation ensures a linear mapping of the pixels to the work surface.

Modulation requires translation between the Cartesian coordinate system and the hybrid coordinate system used by the printhead. For additive manufacturing, this information is compiled by slicing the design into layers then rendering each layer with arcs. The data for each layer are saved in an array; layer rendering information is contained in a two-dimensional array $\operatorname{Arc}[i][j]$ where $i$ is the arc number and $j$ represents a pixel within the arc.

The modulation is performed by turning the laser beam on or off with a set time interval between the voxels. For precise control of energy deposition, the laser energy output can be modulated by pulse width and/or analog intensity for each voxel.

\section{Temperature Prediction}

The LFOS deposits equal energy for any voxel across the work surface, and the laser beam is vertical at all times. The raster method further helps to obtain a uniform temperature distribution across the work surface as the LFOS deposits the same amount of energy for each raster arc. Models such as that of Mirkoohi ${ }^{7}$ can be easily adapted as the input parameters fit those of the Mirkoohi model, such as constant speed and vertical beam.

\section{Energy Density}

In this section, we calculate the volumetric energy density $\mathrm{E}_{\mathrm{v}}$ (Eq. 2) as a function of $\beta$, the angular deflection from the center of the arc. The beam is always perpendicular to the work surface, and the beam's speed and diameter are constant. However, as $\beta$ increases, the spacing between the arcs, which is the effective hatch distance for the LFOS, decreases and the total pixels per area grows (Fig. 7).

The hatch distance can be expressed as

$$
\text { Hatch }=D \cdot \cos (\beta)
$$

Alternatively, it can be expressed as a function of pixel location $i$, where $P$ is the total number of pixels in a full circle:

$$
\text { Hatch }=D \cdot \cos (i / P \cdot 2 \pi) .
$$

Using Eq. 2 for energy density $E_{v}$ but substituting the hatch distance in Eq. 36 for the beam diameter and Eq. 33 for the beam speed yields

$$
E v_{i}=\frac{P_{1}}{v \cdot L_{h} \cdot \operatorname{Def}_{i}}=\frac{P_{1}}{v \cdot L_{h} \cdot D \cdot \cos \left(\frac{i}{P} 2 \pi\right)}
$$



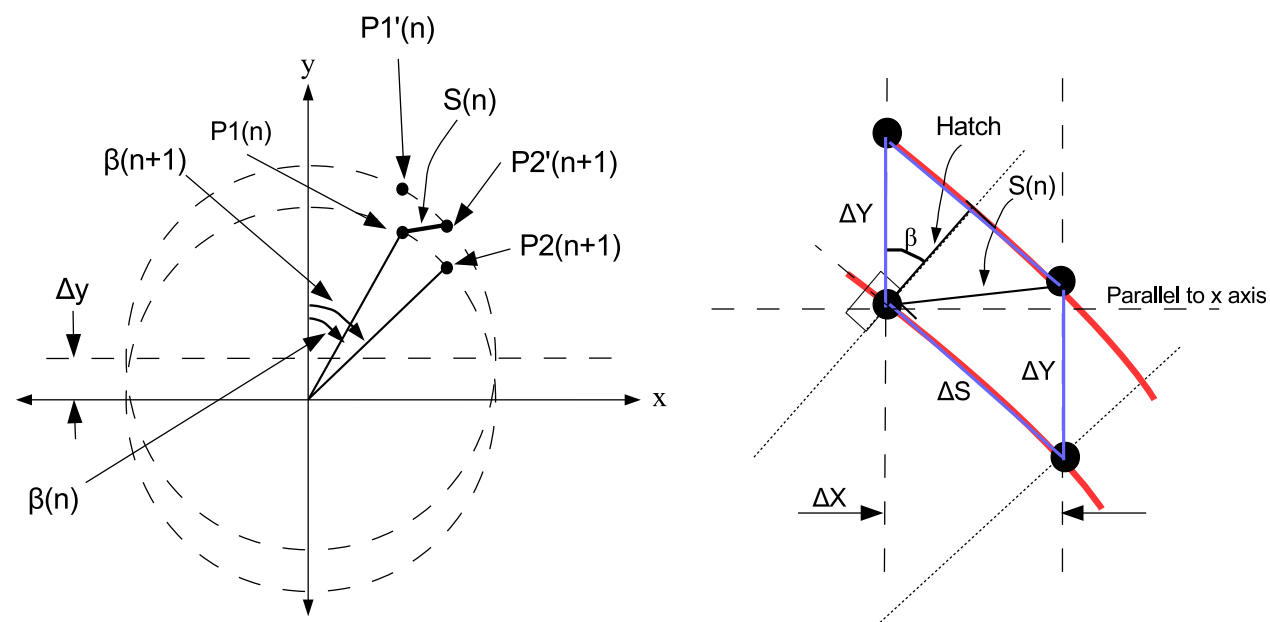

Fig. 7. $\mathrm{P} 1(n)$ and $\mathrm{P}^{\prime}(n+1)$ proximity.

$$
E v_{i}=\frac{P_{1}}{2 \cdot \pi \cdot R \cdot f \cdot L_{h} \cdot D \cdot \cos \left(\frac{i}{P} 2 \pi\right)}=E v_{0} \cdot \frac{1}{\cos \left(\frac{i}{P} 2 \pi\right)}
$$

Alternatively, expressed as a function of $\beta$, we have

$$
E v(\beta)=E v_{0} \cdot \frac{1}{\cos (\beta)}
$$

Using an alternative method to calculate the energy density as power per unit area, where the unit area is the parallelogram defined by the four neighboring pixels (as shown in Fig. 7), we obtain the same result (Eqs. 37-39) (Fig. 8).

As an example from Eqs. 37-39 and the graph above, an opening of $\pm 30^{\circ}$ will increase the energy density and pixels per area by $14 \%$ at the end of the arcs.

\section{Methods for Maintaining Uniform Energy Density}

\section{Print Strategy 1}

The LFOS compensates for this issue by delivering energy per pixel according to its location on the arc, as quantified by Eq. 40:

$$
P l_{i}=P l_{0} \cdot \cos (i / P \cdot 2 \pi)
$$

The power for each pixel is adjusted utilizing Eq. 40. where the modulator can apply either a smaller $P l$ signal (amplitude) or decrease the exposure time for the pixel.

\section{Print Strategy 2}

The slicing algorithm will slice each layer to ensure that $S_{i}$ is adjusted to match Hatch :

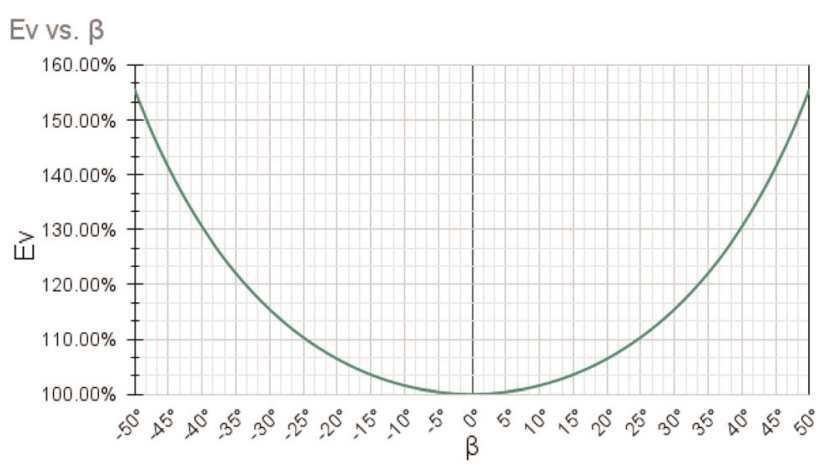

Fig. 8. The Øgon LFOS energy intensity as a function of $\beta$, the arc opening.

$$
\begin{gathered}
\Delta A_{0}=\Delta A_{i}=\Delta S_{i} \cdot \text { Hatch }_{i} \\
\Delta S_{i}=\Delta A_{0} / \text { Hatch }_{i} \\
\Delta S_{i}=\frac{2 \cdot \pi \cdot R \cdot \frac{1}{P}}{\cos \left(\frac{i}{P} \cdot 2 \cdot \pi\right)}
\end{gathered}
$$

In this strategy, the laser power is kept constant so that the burden is on the slicing software.

\section{AM for Metals}

In metal printing, the minimum hatch is determined by the material type and $P l_{i}$ at the arc edges. Alternatively, $S(i)$, the shortest distance between two pixels, can be used to determine the maximum arc opening.

The distance $S(n)$ is calculated by

where $R$ is the arc radius, $D$ is the desired hatch 


$$
S(n)=\sqrt{\left(R \cdot \sin \left(\frac{(n+1)}{P} \cdot 2 \cdot \pi\right)-R \cdot \sin \left(\frac{n}{P} \cdot 2 \cdot \pi\right)\right)^{2}+\left(\mathrm{D}+\mathrm{R} \cdot \cos \left(\frac{(\mathrm{n}+1)}{\mathrm{P}} \cdot 2 \cdot \pi\right)-\mathrm{R} \cdot \cos \left(\frac{\mathrm{n}}{\mathrm{P}} \cdot 2 \cdot \pi\right)\right)^{2}}
$$

distance, $n$ is the pixel location on the present arc, and $n+1$ is the neighboring pixel on the next arc.

Equations 45 and 46 is a good approximation for $S$ (i) because $R \gg D$.

$$
\begin{gathered}
S(\beta)=\text { Hatch } / \cos (90-\beta)=D \cdot \cos (\beta) / \sin (\beta)=D / \tan (\beta) \\
S(i)=\text { Hatch } / \cos (\pi / 4-i / P \cdot 2 \cdot \pi)=D / \tan (2 \cdot \pi \cdot i / P)
\end{gathered}
$$

\section{Repeatability}

A typical repeating error for the linear conveyor used for the initial prototype is $5 \mu \mathrm{m}$ for every 100 $\mathrm{mm}$. The weak point in the polar system is the motor because tilt from the vertical axis will affect the positioning. The tilt is addressed by keeping the center of mass on the rotational axis.

\section{Stability}

The M1 concave surface structure shifts the center of mass from the actuator rotational axis. The M1 reflector is carried by a jacket stabilizer to keep the center of mass along the optical axis, hence overcoming any motor drift if it exists (Fig. 9).

\section{RESULTS AND DISCUSSION}

The AM industry has attempted to mitigate -related errors by using several methods, each of which reduces the maximum value of theta required for the work surface.

One method is to increase the distance from the GS to the work surface. However, this results in bulky printers that make the design of the optics more challenging, because they have increased sensitivity to noise and magnified offset/drift errors.

Another popular method to reduce the maximum value of is to use multiple lasers working in parallel. This obviously reduces print time. However, it also increases the complexity of the preprint algorithms for two reasons. First, they must calculate multiple print paths, one for each laser. Second, the stitch lines between adjacent lasers' print areas lie at the edge of each laser's range, where all the theta-related errors discussed above are at their maximum. Complex engineering and computational work is required to ensure that the voxels on the stitch lines are correctly aligned and receive the correct amount of energy.
Metal printing requires a high degree of temperature uniformity across the whole print surface. The GS needs to address multiple causes to mitigate the heat nonuniformity. A LFOS, on the other hand, eliminates the major causes of the problem. Additionally, because GS printers require a tall print chamber to keep the angle of incidence low, it is harder for them to control the chamber temperature. The design of the LFOS as embodied in the Tecnica Øgon permits a very short chamber height.

\section{CONCLUSION}

The LFOS concept solves the galvanometer scanners' fundamental problem of inconsistent energy deposition. While the surface beam speed, optical path length, and angle of incidence (which affect the beam size and shape) vary as the galvanometers scan the work surface, these properties remain constant for LFOS (Table 1).

The $y$-dimension of the LFOS print area can be increased by using a longer linear actuator. Although the $x$-dimension is limited by the size of the M2 mirror, the print head is portable so that it can be mounted on a robot to cover a larger print area in a sequence of stripes. This kind of scanning would have a repeatability error of $50 \mu \mathrm{m}$ for a typical

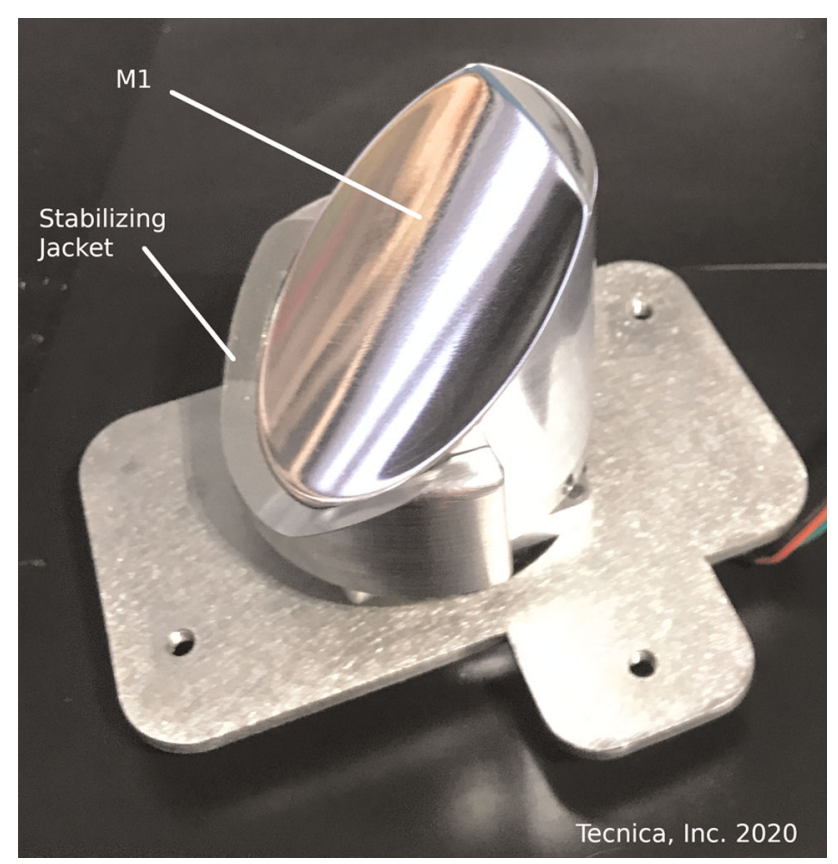

Fig. 9. M1 with stabilizing jacket mounted on a motor. 
Table 1. Head-to-head comparison between the GS and the Øgon LFOS

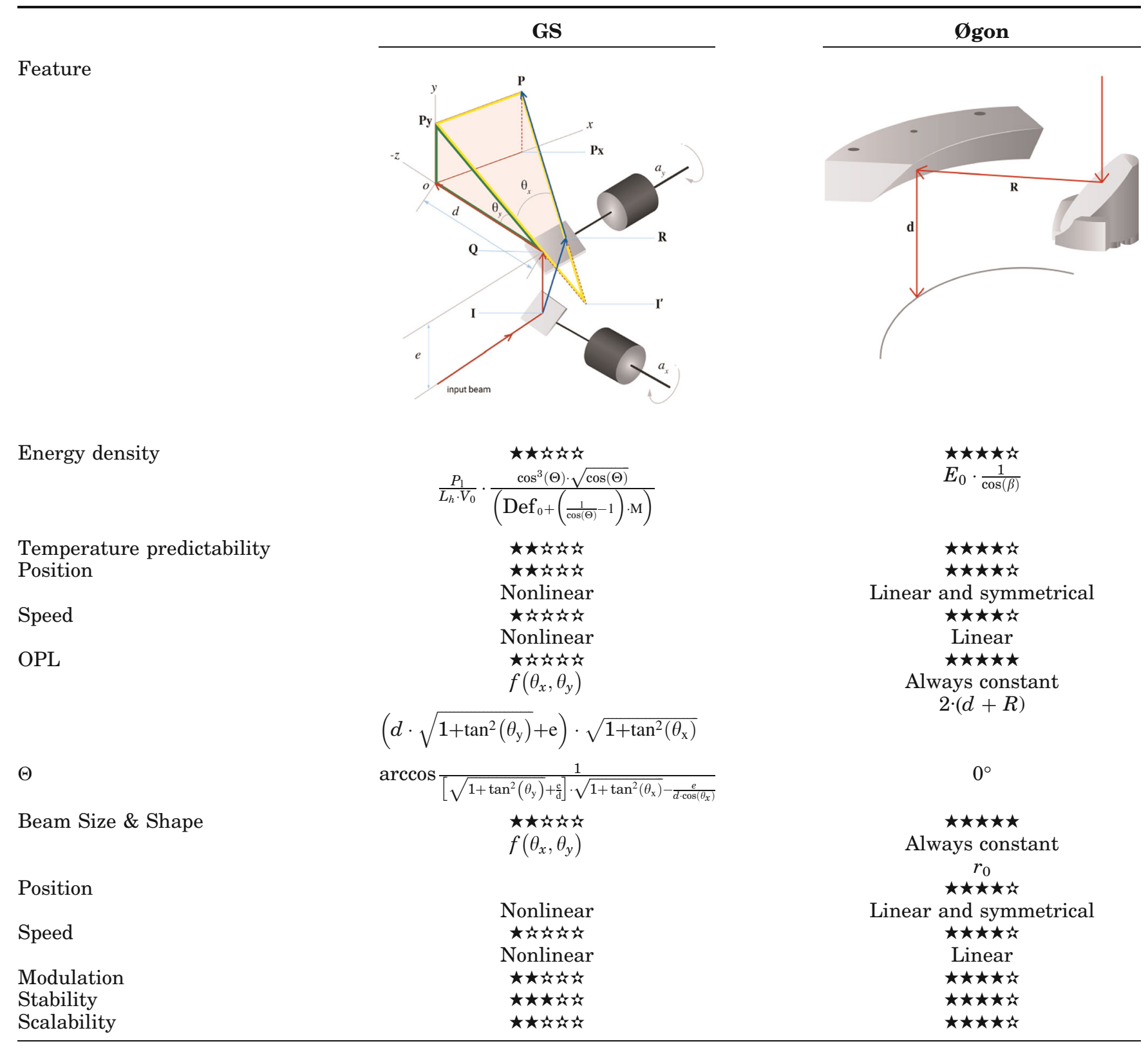

robot with a 2-m arm. This is an easy adaptation of existing workshops utilizing their robots.

The LFOS laser energy output can be modulated by pulse width and/or analog intensity, and the scanner consistently delivers $100 \%$ of that energy to each pixel across the entire work surface. This precise control of energy deposition and surface temperature makes it the ideal platform for developing new algorithms for additive manufacturing metal printing.

It would be interesting to use an LFOS to experimentally validate some of the temperature prediction models for metal printing. For example, the Øgon LFOS embodiment modulator could incorpo- rate the Mirkoohi model ${ }^{7}$ coupled with machine learning to predict the $z$-axis of the melt pool, which would provide superior temperature prediction.

\section{ACKNOWLEDGEMENTS}

The author thanks Debora Rozenblum and Gabriel Goodman for their invaluable contributions to creating this manuscript.

\section{OPEN ACCESS}

This article is licensed under a Creative Commons Attribution 4.0 International License, which permits use, sharing, adaptation, distribution and reproduction in any medium or format, as long as you 
give appropriate credit to the original author(s) and the source, provide a link to the Creative Commons licence, and indicate if changes were made. The images or other third party material in this article are included in the article's Creative Commons licence, unless indicated otherwise in a credit line to the material. If material is not included in the article's Creative Commons licence and your intended use is not permitted by statutory regulation or exceeds the permitted use, you will need to obtain permission directly from the copyright holder. To view a copy of this licence, visit http://creative commons.org/licenses/by/4.0/.

\section{CONFLICT OF INTEREST}

Charles Bibas reports the following details of affiliation or involvement in an organization or entity with a financial or nonfinancial interest in the subject matter or materials discussed in this manuscript: The author is the CEO and Founder of Tecnica, Inc., a corporation that is utilizing the lens-free optical scanner (LFOS) in 3D printing technology. The author is also the inventor of the LFOS.

\section{REFERENCES}

1. G.D. Goh, S.L. Sing, and W.Y. Yeong, Artif. Intell. Rev. 54, 63. https://doi.org/10.1007/s10462-020-09876-9 (2021).

2. X. Qi, G. Chen, Y. Li, X. Cheng, and C. Li, Addit. Manuf. Rev. 5, 721. https://doi.org/10.1016/j.eng.2019.04.012 (2019).

3. Z. Li, Z. Zhang, J. Shi, and Wu. Dazhong, Rob. Comput. Integr. 57, 488. https://doi.org/10.1016/j.rcim.2019.01.004 (2019).

4. R. Goodridge, and S. Ziegelmeier, Laser Additive Manufacturing (Woodhead, Cambridge, 2017), pp 181-204.

5. M. Nozar, I. Zetková, O. Hronek, in Paper presented at the 29th DAAM International Symposium of intelligent manufacturing and automation, Vienna University of Technology,
Austria, 24-27 October 2018. https://doi.org/10.2507/29th. daaam.proceedings.113.

6. U.S. Bertoli, A. Wolfer, M.J. Matthews, J. Delplanque, and J.M. Schoenung, Mater. Des. 113, 331. https://doi.org/10. 1016/j.matdes.2016.10.037 (2017).

7. E. Mirkoohi, J. Ning, P. Bocchini, O. Fergani, K.-N. Chiang, and S.Y. Liang, J. Manuf. Mater. Proc. 2, 63. https://doi.org/ 10.3390/jmmp2030063 (2018).

8. N. Benoit, H. Rana, A. Valamanesh, Applying machine learning for real time optimization of powder bed manufacturing. Worcester Polytechnic Institute (Worcester Polytechnic Institute, 2019), https://digital.wpi.edu/show/ 2514np09z. Accessed November 4, 2021.

9. R. Wauthle, B. Vrancken, B. Beynaerts, K. Jorissen, J. Schrooten, J.-P. Kruth, and J. Van Humbeeck, Addit. Manuf. 5, 77. https://doi.org/10.1016/j.addma.2014.12.008 (2015).

10. L. Song, V. Bagavath-Singh, B. Dutta, and J. Mazumder, Int. J. Adv. Manuf. Technol. 58, 247. https://doi.org/10.1007/ s00170-011-3395-2 (2012).

11. A.S. Metel, M.M. Stebulyanin, S.V. Fedorov, and A.A. Okunkova, Rapid Manuf. Technol. 7, 5. https://doi.org/10. 3390/technologies7010005 (2019).

12. H. Jigang, Q. Qin, W. Jie, and F. Hui, Int. J. Mater. Mech. Manuf. 6, 332. https://doi.org/10.18178/ijmmm.2018.6.5.402 (2018).

13. C. Bibas, A. Øgon, Revolutionary new lens free optical scanner (LFOS) for additive manufacturing (AM". Paper presented at the 32nd International Solid Freeform Fabrication Symposium, University of Texas, Austin, 2-4 Aug 2021.

14. M. Xianyu, C. Guohua, L. Xue, and L. Qiongying, J. Phys. Conf. Ser. 1345, 022068. https://doi.org/10.1088/1742-6596/ 1345/2/022068 (2019).

15. W. Zhang, W. Guo, C. Zhang, and S. Zhao, Sensors 19, 1353. https://doi.org/10.3390/s19061353 (2019).

16. V.F. Duma, Appl. Math. Model. 67, 456. https://doi.org/10. 1016/j.apm.2018.11.001 (2018).

17. C. Bibas, Beam director with improved optics. US Patent 10,416,444, (2019).

18. C. Bibas, D.P. Hugo, N. Zhou, Beam director. US Patent 9,435,998, (2016).

Publisher's Note Springer Nature remains neutral with regard to jurisdictional claims in published maps and institutional affiliations. 